\title{
Scintillation measurements at Bahir Dar during the high solar activity phase of solar cycle 24
}

\author{
Martin Kriegel $^{1}$, Norbert Jakowski ${ }^{1}$, Jens Berdermann ${ }^{1}$, Hiroatsu Sato ${ }^{1}$, and Mogese Wassaie Mersha ${ }^{2}$ \\ ${ }^{1}$ German Aerospace Center (DLR), Institute of Communications and Navigation, Kalkhorstweg 53, \\ 17235 Neustrelitz, Germany \\ ${ }^{2}$ Washera Geospace and Radar Science Laboratory, Bahir Dar University, Bahir Dar, Ethiopia \\ Correspondence to: Martin Kriegel (martin.kriegel@dlr.de)
}

Received: 22 July 2016 - Revised: 29 November 2016 - Accepted: 2 December 2016 - Published: 13 January 2017

\begin{abstract}
Small-scale ionospheric disturbances may cause severe radio scintillations of signals transmitted from global navigation satellite systems (GNSSs). Consequently, smallscale plasma irregularities may heavily degrade the performance of current GNSSs such as GPS, GLONASS or Galileo. This paper presents analysis results obtained primarily from two high-rate GNSS receiver stations designed and operated by the German Aerospace Center (DLR) in cooperation with Bahir Dar University (BDU) at $11.6^{\circ} \mathrm{N}, 37.4^{\circ} \mathrm{E}$. Both receivers collect raw data sampled at up to $50 \mathrm{~Hz}$, from which characteristic scintillation parameters such as the S4 index are deduced.

This paper gives a first overview of the measurement setup and the observed scintillation events over Bahir Dar in 2015. Both stations are located close to one another and aligned in an east-west, direction which allows us to estimate the zonal drift velocity and spatial dimension of equatorial ionospheric plasma irregularities. Therefore, the lag times of moving electron density irregularities and scintillation patterns are derived by applying cross-correlation analysis to high-rate measurements of the slant total electron content (sTEC) along radio links between a GPS satellite and both receivers and to the associated signal power, respectively. Finally, the drift velocity is derived from the estimated lag time, taking into account the geometric constellation of both receiving antennas and the observed GPS satellites.
\end{abstract}

Keywords. Ionosphere (ionospheric disturbances; ionospheric irregularities; instruments and techniques)

\section{Introduction}

It is generally agreed that localized depletions of the lowlatitude $\mathrm{F}$ region electron density may be generated due to the Rayleigh-Taylor plasma instability after sunset (Kelley, 2009). The plasma density inside these irregularity regions is strongly reduced, thus forming equatorial plasma bubbles (EPBs) that together with electron density irregularities cause diffraction and forward-scattering of transionospheric radio signals; this is known as scintillation (Basu and Basu, 1981; Valladares et al., 2004). When the plasma irregularities are fully developed, the irregular structures, causing scintillation of satellite signals, are expected to flow along the ambient zonal drift motion. The $\boldsymbol{E} \times \boldsymbol{B}$ zonal drift moves the plasma perpendicularly to the ambient horizontal magnetic field and the downward-directed electric field that is mainly generated by thermospheric winds in the lowlatitude ionosphere (Kelley, 2009). The drift motions have diurnal variations in flow direction and magnitude, but in general the zonal drift flows westward in the daytime and eastward in the nighttime. The peak eastward drift speed in the low-latitude pre-midnight sector has a typical magnitude of $100 \mathrm{~m} \mathrm{~s}^{-1}$, while daytime drifts usually peak at $40 \mathrm{~m} \mathrm{~s}^{-1}$ in the quiet-time ionosphere (Fejer et al., 1985, 1991). From GPS L1 signals, Kil et al. (2000) derived eastward velocities of ionospheric irregularities of $50-100 \mathrm{~m} \mathrm{~s}^{-1}$ at midnight in the South American sector for magnetically quiet days.

Although the occurrence of eastward drift irregularities is expected in the low-latitude African sector, their existence has been shown only in a few cases (e.g. Wiens et al., 2006). Using a single global navigation satellite system (GNSS) station, Hlubek et al. (2014) have recently 
shown statistics of scintillation events occurring over the Bahir Dar region for GPS frequencies L1, L2C, and L5, GLONASS L1 and L2, and Galileo E1 and E5, covering the year 2013. These frequencies range between $1176.45 \mathrm{MHz}$ (Galileo E5) and $1610.485 \mathrm{MHz}$ (GLONASS L1). The obtained results are based on high-rate real-time GNSS measurements recorded at the DLR's msbd01 station (see Fig. 1) in Bahir Dar, Ethiopia. It has been shown that the L5/E5a frequency is much more strongly affected by scintillations compared to the L1/E1 frequency. Overall GLONASS L2 shows the largest sensitivity with respect to scintillation events. Additionally, these studies also confirm the daily and seasonal variability of scintillation events in low-latitude regions reported by Wiens et al. (2006) and Alfonsi et al. (2013).

In this work we apply a multi-station analysis to study the characteristics of plasma irregularities, including their zonal drift characteristics. Therefore, this paper first describes both station set-ups and their capabilities to detect scintillation events. Afterwards, we give a short review of simultaneously observed scintillation events in 2015. This includes the analysis of our scintillation processor output compared to external data from a nearby GNSS station operated by the Technical University of Berlin (TUB). Finally, we estimate the zonal drift velocity and spatial dimension of plasma irregularities over Bahir Dar using a cross-correlation analysis method, which is applied to high-rate data from DLR's GNSS network.

\section{GNSS station set-up}

Since 2010 DLR has established two high-rate GNSS receiver stations in close cooperation with Bahir Dar University for monitoring and analysing amplitude scintillations in equatorial regions.

The first station, msbd01, was initially deployed in 2010 at Bahir Dar University (Peda Campus). It operates a Javad Delta G3T receiver combined with an external Temex LPFRS (low-profile frequency Rb standard) rubidium clock and a Javad RingAnt-G choke ring antenna to minimize multipath effects. To intensify scintillation research in equatorial regions and as a result of the cooperation with Bahir Dar University the second station, msbd02, was deployed 2014 at the Yibab Campus (see Fig. 1), which is located around $7 \mathrm{~km}$ westward of msbd01. The slightly improved hardware and software set-up operates a JAVAD Delta 3G GNSS receiver in combination with an external Temex LPFRS rubidium clock and a Leica AR25 choke ring antenna. After the ISEA14 conference in 2015 also msbd01 was modernized to harmonize the level of performance and robustness. Both JAVAD receivers are configured to track GPS (L1, L2, L5), GLONASS (L1, L2), Galileo (E1, E5a) and Beidou as well as geostationary satellites of space-based augmentation systems like the Wide Area Augmentation System (WAAS) and the European Geostationary Navigation Overlay Service

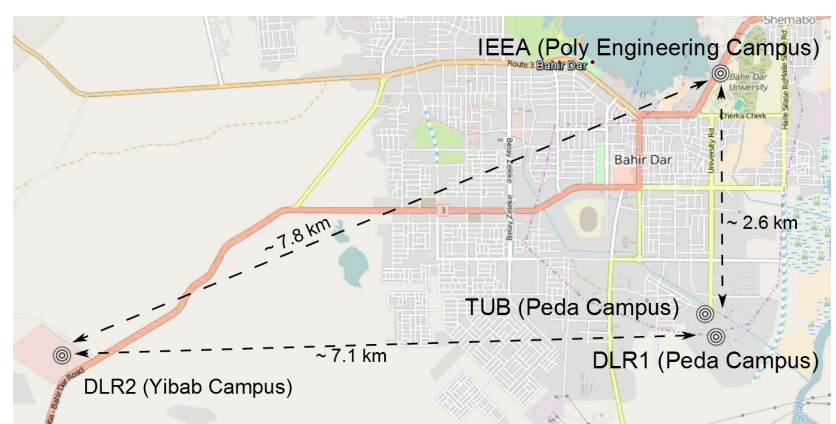

Figure 1. Geographic distribution of high-rate GNSS receivers in Bahir Dar, Ethiopia.

(EGNOS) with a sampling rate of $50 \mathrm{~Hz}$. Since power outages occur frequently in Ethiopia, both stations are plugged to uninterruptible power supply (UPS) devices in order to bridge power outages of up to $7 \mathrm{~h}$ which is adequate for $95 \%$ of all outages.

To simplify the station maintenance and to enable distributed real time and post processing of the high-rate GNSS data for scintillation research, both stations are integrated into the EVnet (Experimentation and Verification Network) architecture of DLR (Noack et al., 2005). Over the past years both systems were able to continuously record highrate GNSS raw data, providing an important dataset for ongoing studies on amplitude scintillations in equatorial regions.

In collaboration with the Technical University of Berlin and the French company IEEA, two additional high-rate GNSS station were deployed. The TUB station uses a Septentrio PolaRx GNSS receiver and is located at the Peda Campus near the msbd01 station (Čokrlić and Galas, 2013). The IEEA station operates a Novatel GSV4004B GISTM and is located at the Poly Engineering Campus, roughly $2.6 \mathrm{~km}$ north of the Peda Campus. All together these four stations, as illustrated in Fig. 1, form an unique local network to study amplitude scintillations.

\section{Monitoring of amplitude scintillations}

Both DLR stations were designed to monitor total electron content (TEC) and signal strength scintillations on GNSS signals in near real time. Amplitude scintillations are rapid irregular variations in the signal amplitude (e.g. signal fading and scattering) due to the spatially and temporally varying density of free electrons on a very small scale throughout the different layers of the ionosphere. To detect whether GNSS signal is affected by amplitude scintillations, the variation in the signal intensity $I$ is observed and quantified by the widely used $S_{4}$ index, which is the standard deviation of the received signal intensity $I$ divided by its mean value (for normalization). In general the $S_{4}$ index is calculated per GNSS link by using a moving window in the time domain with a window width of $60 \mathrm{~s}$ 
Table 1. Classification of amplitude scintillation events.

\begin{tabular}{ll}
\hline Magnitude of $S_{4}$ & Classification of scintillation event \\
\hline$S_{4}>0.3$ & moderate \\
$S_{4}>0.6$ & strong \\
$S_{4}>0.9$ & extreme
\end{tabular}

The monitoring of $S_{4}$ derived from the DLR's worldwide distributed EVnet stations showed that $S_{4}$ values up to 0.3 are mostly caused by the natural noise of the signal intensity.

$S_{4}=\sqrt{\frac{\left\langle I^{2}\right\rangle-\langle I\rangle^{2}}{\langle I\rangle^{2}}}$

where \langle\rangle denotes the corresponding average value. We derive $S_{4}$ measurements according to Eq. (1) from high-rate GNSS data streams $(50 \mathrm{~Hz})$ in real-time $(1 \mathrm{~min}$ update rate) and in post-processing mode, since the operational JAVAD receivers including JAVAD's software tools do not provide $S_{4}$ directly. The signal intensity $I$ can be derived from the signal amplitude $A\left(I \propto A^{2}\right)$. The scintillation-processing software calculates the signal amplitude $A$ according to

$A^{2}=i^{2}+q^{2}$.

The high-rate inphase $(i=A \cdot \cos \Phi)$ and quadrature $(q=$ $A \cdot \sin \Phi)$ components are provided by the GNSS receiver (JAVAD, 2015, p. 88). The magnitude of $S_{4}$ is limited within the range $\left\{S_{4} \in \mathbb{R} \mid 0 \leq S_{4} \leq \sqrt{2}\right\}$ when assuming a Nakagami distribution as a fading model for GNSS signal fading (Hegarty et al., 2001). In order to rate multiple $S_{4}$ measurements, we classify scintillation events by their magnitude as listed in Table 1.

The interpretation of the magnitude of $S_{4}$ should incorporate the spatial geometry of the GNSS link, since it is correlated with the elevation angle of the observed GNSS satellite. If the elevation is very low, the propagation time through potentially perturbed regions of the ionosphere increases, with the consequence that the occurrence of scintillation events during low-elevation scenarios is naturally higher than at high-elevation scenarios. Also, multipath effects due to very low-elevation scenarios or physical obstacles may degrade the signal quality and can lead to false assumptions on ionosphere-generated amplitude scintillations. We did not quantify these effects but systematically try to exclude them by only considering observations at elevations greater than $20^{\circ}$. Based on the geographical receiver location, there might also be a dependency on the azimuth of the observed GNSS link. The receivers in Bahir Dar are located between the northern and southern crest anomaly regions. As Fig. 2 shows, GNSS measurements with azimuth angles approximately in the north-south direction and vice versa are naturally much more affected by amplitude scintillation events than measurements in azimuthal east-west di-
Table 2. Scintillation events recorded by both DLR stations in 2015 .

\begin{tabular}{lcl}
\hline Month & Affected days & Activity/relevance \\
\hline February & 3 & moderate \\
March & 6 & strong \\
April & 9 & strong \\
May & 7 & moderate \\
December & 1 & moderate \\
\hline \multicolumn{2}{l}{ The gap from May to December is due to the outage of msbd01 }
\end{tabular}
before its modernization in October 2015.

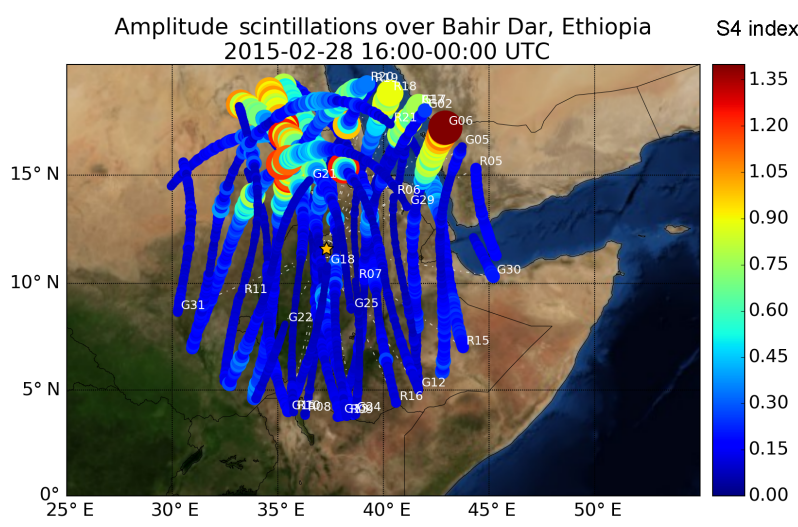

Figure 2. $S_{4}$ estimated from $50 \mathrm{~Hz}$ GNSS data recorded between 16:00 and 00:00 UTC (LT $-3 \mathrm{~h}$ ) on 28 February 2015 for msbd02.

rection. The strong scintillation activity around $14-19^{\circ} \mathrm{N}$ obviously fits well with the steep TEC gradient associated with the northward crest of the equatorial anomaly (e.g. Muella et al., 2010).

In order to select appropriate scintillation events for the estimation of equatorial plasma bubble characteristics over Bahir Dar, all available datasets obtained in 2015 were scanned for identifying and classifying scintillation events by applying Table 1. Although there were many events at each DLR station, only a few events having small data gaps could be simultaneously recorded at both receivers, as shown in Table 2. As is well known (e.g. Basu and Basu, 1981; Aarons, 1982; Hlubek et al., 2014), signal scintillations could be observed in the evening hours after sunset.

To prove the plausibility of the detected scintillation events we compared DLR data with data collected by TUB in the same time periods at the most significant events.

Figure 3 shows an example of a typical scintillation event which was simultaneously recorded independently of the stations msbd01, msbd02 and tubbd01 (see Fig. 1) on 28 February 2015. It shows enhanced signal intensity variations on multiple GNSS links within the L1 band $(1575.42 \mathrm{MHz})$. Also, the link-based signature of $S_{4}$ evolving over time derived by DLR and TUB software reveals a high similarity as shown for GPS satellite G24 in Fig. 4. This proves the fact that both systems are capable of properly detecting amplitude scintillation events for correlation studies. 

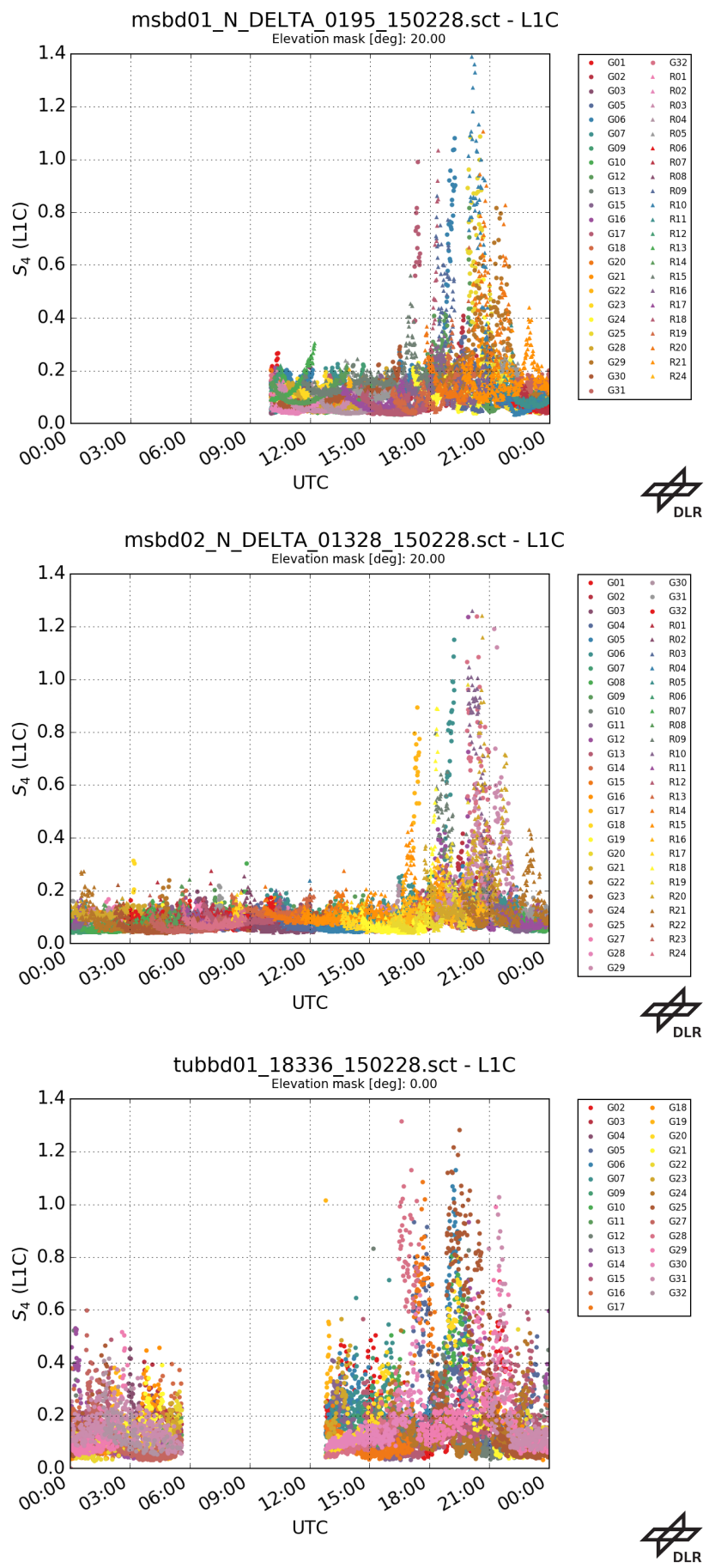

Figure 3. Typical amplitude scintillation event after sunset recorded at both DLR stations and the TUB station on 28 February 2015 over Bahir Dar, Ethiopia.

\section{Characterization of plasma irregularities}

Both DLR stations, msbd01 and msbd02, are located at different longitudes but at nearly the same latitude and simultaneously record signals from the same GPS satellite at a sam-
S4 comparision for 2015-02-28 / G24 in Bahir Dar, Ethiopia
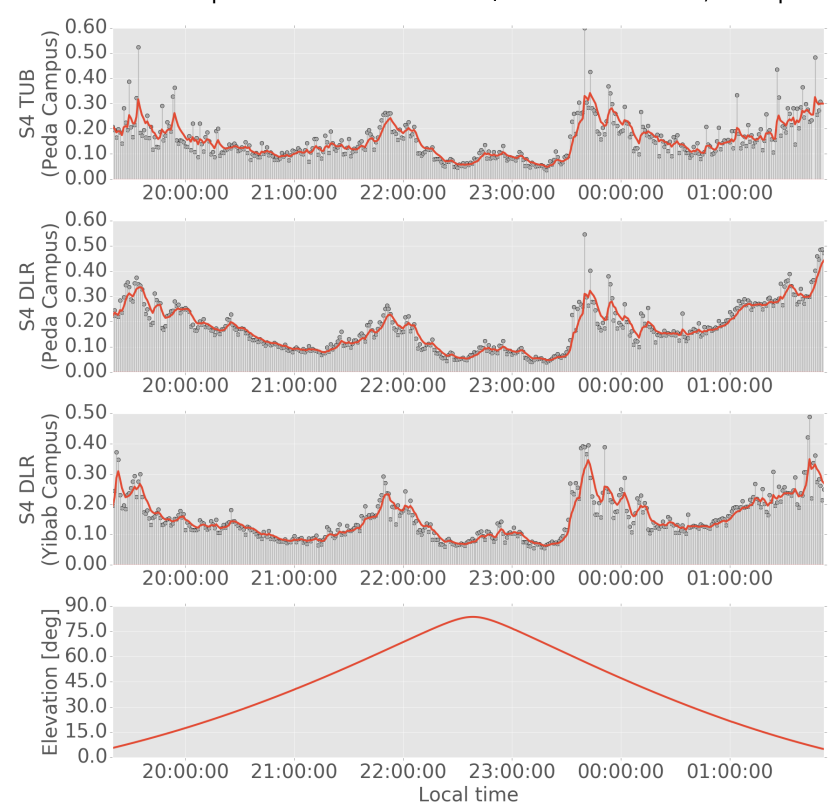

Figure 4. Signature (red line, exponentially-weighted moving average, window size: $3 \mathrm{~min}$ ) of $S_{4}$ indices (grey dots) calculated for satellite G24 from different scintillation processors in comparison to the averaged elevation (below).

pling rate of $50 \mathrm{~Hz}$. Figure 5 illustrates the overall measurement principle for characterizing plasma irregularities from high-rate GPS measurements.

After sunset, equatorial plasma irregularities begin to evolve, thus causing radio scintillations associated with, e.g., enhanced signal power variability and deep fading effects when the ray path enters the first Fresnel zone (Hargreaves, 1992, p. 33). Assuming that electron density irregularities are well established at a height of about $h_{\mathrm{I}}=350 \mathrm{~km}$, the first Fresnel $(n=1)$ zone of GPS L1 signals $(\lambda=19.042 \mathrm{~cm})$ in zenith direction $\left(d_{1} \simeq h_{\mathrm{I}}, d_{2} \simeq 20850 \mathrm{~km}\right)$ has a radius of $r_{\mathrm{F}} \approx 256 \mathrm{~m}:$

$r_{\mathrm{F}}=\sqrt{\frac{n \lambda d_{1} d_{2}}{d_{1}+d_{2}}}$

where $d_{1}$ is the distance between the ionospheric pierce point (IPP) and the GPS satellite and $d_{2}$ is the distance between the GNSS receiver and the related IPP according to Fig. 5. Scintillation and TEC depletion patterns can be observed by monitoring the signal power and the slant total electron content (sTEC) over time. In Figs. 6 and 7, measurements of the satellite G24 for both DLR stations obtained on 28 February 2015 are shown. The depletion signature starts developing when the irregularity region enters the first Fresnel zone, reaches its maximum depletion at the highest Fresnel zone coverage and decreases when the bubble region leaves the first Fresnel zone. 


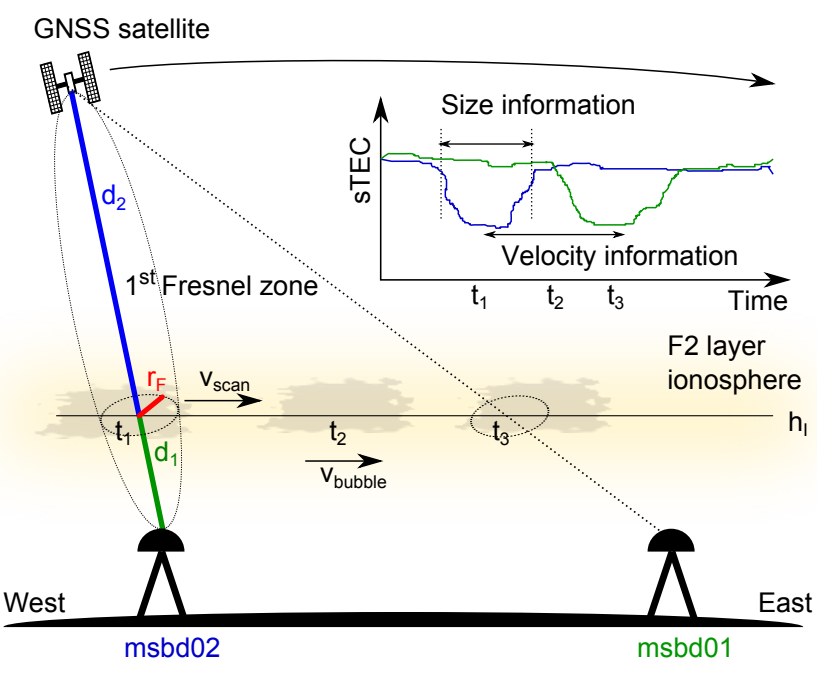

Figure 5. Scheme of GNSS link-based estimation of plasma bubble characteristics from multiple high-rate GNSS stations in Bahir Dar.

Since the horizontal component of the plasma irregularity drift is a zonal motion, irregularity effects should be visible at both links with a similar signature but shifted in time. Analogously to Wang and Morton (2015), the overall irregularity drift velocity, $v_{\lambda}$, can be estimated from the irregularity pattern velocity, $v_{\lambda \text { PATTERN }}$, and the scanning velocity, $v_{\lambda I P P}$ :

$v_{\lambda}=v_{\lambda \text { PATTERN }}+v_{\lambda I P P}$.

$v_{\lambda}$ can be estimated by determining the time lag of the irregularity pattern observed on the same links from both stations and its distance. $v_{\lambda I P P}$ is derived from the dynamically changing geometry of both observing GNSS links. According to Kil et al. (2000) and Figs. 5 and 6, the estimated plasma drift velocity $v_{\lambda}$ can be used to derive the zonal dimension $d_{\lambda}$ of the irregularity region by multiplying the velocity with the temporal width of the depletion signature according to

$d_{\lambda}=v_{\lambda} \cdot\left(t_{\mathrm{e}}-t_{\mathrm{s}}\right)$,

with $t_{\mathrm{s}}$ as starting time and $t_{\mathrm{e}}$ as end time of the depletion signature in seconds.

To estimate the drift velocity of the plasma irregularity patterns, the cross-correlation analysis is applied on two different link-based time series in order to estimate the time lag of the irregularity patterns between both stations as initially developed by Briggs et al. (1950) and Briggs (1968). The first series involves the scintillation index $S_{4}$, calculated according to Eq. (1) for a window length of $1 \mathrm{~min}$, but the window is advanced by the native data rate of $50 \mathrm{~Hz}$. The second series involves the relative sTEC derived from $50 \mathrm{~Hz}$ carrier phase measurements $\Phi_{n}$, where $f_{n}$ and $\lambda_{n}$ denote the signal's frequency and wavelength and $K=40.3 \mathrm{~m}^{3} \mathrm{~s}^{-2}$ :

sTEC $=\frac{f_{1}^{2} f_{2}^{2}}{K\left(f_{1}^{2}-f_{2}^{2}\right)}\left(\lambda_{1} \Phi_{1}-\lambda_{2} \Phi_{2}\right)-B$

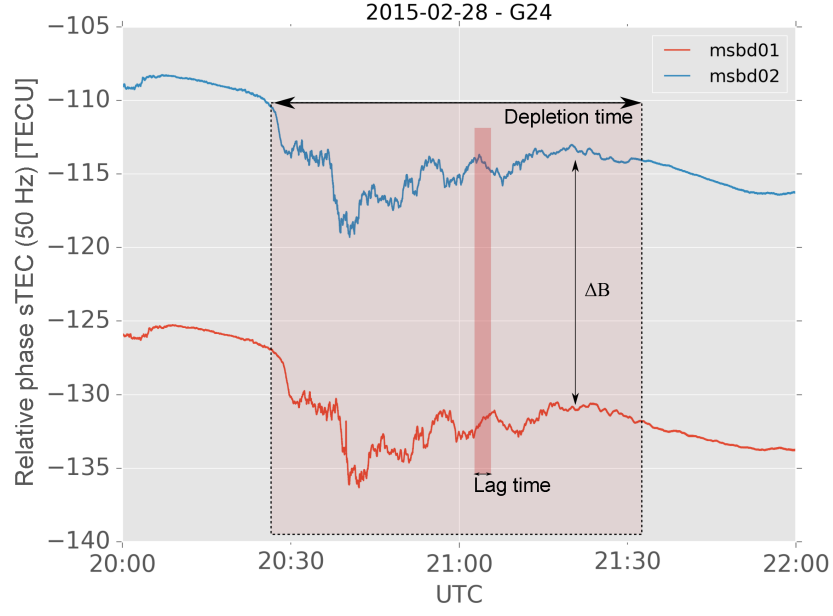

Figure 6. Observed sTEC depletion moving eastward within a time span of $1 \mathrm{~h}$ and a maximum depletion of 10 TEC units for satellite G24 over Bahir Dar, Ethiopia.

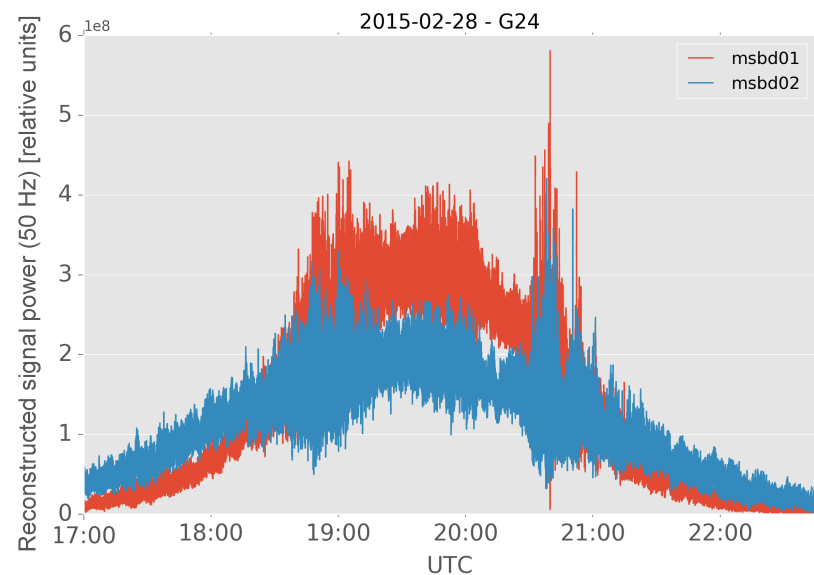

Figure 7. Signal power, $S_{\mathrm{I}}$, derived from inphase and quadrature components. $S_{\text {I }}$ increases with the decreasing distance to the rising satellite and decreases due to the growing distance when the satellite sets.

Both datasets need to be preprocessed before applying them to the cross-correlation analysis. Figure 6 shows a typical example of the estimated relative sTEC derived from Eq. (6) for msbd01 and msbd02. Both time series show a typical depletion scenario of several TEC units over $1 \mathrm{~h}$ with similar sTEC variation, but an offset, $\Delta B=B_{\mathrm{msbd} 01}-B_{\mathrm{msbd} 02}$, of both time series can be observed. The offset $\Delta B$ is composed of the sum of differential phase biases and the thermal noise of both receivers and the observed satellite, integer ambiguities for both signal measurements including possible cycle slips, and multipath effects in low-elevation scenarios. To get optimal results for the cross-correlation analysis, $\Delta B$ is minimized as follows.

Strong scintillations might lead to a loss of lock and cycle slips. Within the sTEC time series, the effect of cycle slips 
can be found as large jumps between consecutive epochs (Hofmann-Wellenhof et al., 2001, p. 186). These jumps are detected by analysing the temporal variation in the sTEC time series. In cases of detected cycle slips, the slip-induced offset is determined and the time series of sTEC are connected by reducing the time series after the slip by this offset.

After cycle slip correction the impact of unknown phase ambiguities has the highest contribution to $\Delta B$. This is due to the different lock times when tracking the satellite. A common practice to approximate the differential phase ambiguities is to adopt a Hatch filter and apply it to low-noise sTEC derived from phase measurements by introducing additional sTEC estimations from pseudorange measurements (Subirana et al., 2013, p. 79). One disadvantage of this procedure is that the P-code-filtered sTEC measurements are affected by a much higher noise level than the initial estimations. The noise introduced strongly depends on the enhanced noise level of the pseudorange measurements and on the length of the smoothing window of the Hatch filter. The differential P code biases can be estimated by using a model-based TEC calibration as used for generating TEC maps provided by the DLR service SWACI in near real time (Jakowski et al., 2011). In the end, the techniques mentioned to minimize $\Delta B$ are intentionally not applied for this analysis because they will degrade the precision of the initial low-noise sTEC derived from carrier phase measurements. They might also change the shape of the irregularity pattern and as a consequence sophisticate the results of the cross-correlation analysis. Since we are predominantly interested in the estimation of the lag of a pattern occurring in two different time series, the knowledge of the absolute sTEC value is not mandatory. To minimize $\Delta B$, both sTEC time series are standardized by simply subtracting their mean behaviour $\langle\mathrm{sTEC}\rangle$ :

$\overline{\mathrm{STEC}}=\mathrm{sTEC}-\langle\mathrm{STEC}\rangle$.

The mean behaviour $\langle\mathrm{sTEC}\rangle$ is modelled by using the exponentially weighted moving average with the smoothing factor $\alpha=0.133 \times 10^{-3}$ that corresponds to a span size $s$ of $5 \mathrm{~min}$ for $50 \mathrm{~Hz}$ data $\left(\alpha=\frac{2}{s+1}\right)$ :

$$
\langle\mathrm{sTEC}\rangle=(1-\alpha) \cdot \mathrm{sTEC}_{t-1}+\alpha \cdot \operatorname{sTEC}_{t} .
$$

The $S_{4}$ series of both stations already shows a similar mean behaviour and variation as plotted in Fig. 8, since the $S_{4}$ index is already a normalized observable. Finally, the lag of the scintillation pattern, $\tau_{S_{4}}$, and the lag of the depletion pattern, $\tau_{\text {STEC }}$, can be estimated by determining the argument of the maximum of the cross-correlation applied to both time series by replacing $S$ with $S_{4}$ or sTEC:

$\tau_{S}=\underset{t_{k}}{\arg \max }\left(\frac{\sum_{k} S_{\mathrm{msbd} 01}\left(t_{k}\right) S_{\mathrm{msbd} 02}\left(\tau-t_{k}\right)}{\sqrt{\sum_{k} S_{\mathrm{msbd} 01}^{2}(t) \sum_{k} S_{\mathrm{msbd} 02}^{2}\left(t_{k}\right)}}\right)$.
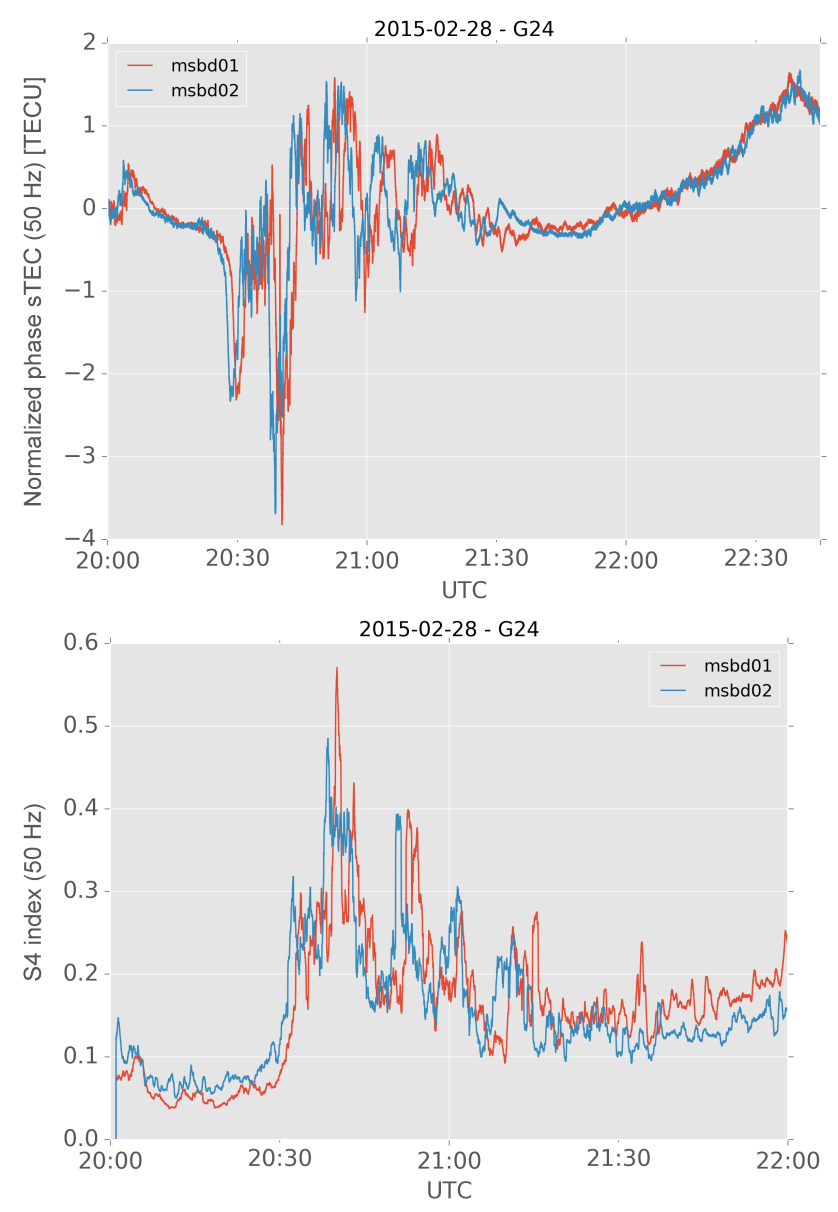

Figure 8. Normalized sTEC and $S_{4}$ measurements for G24 as input for cross-correlation analysis.

Due to the normalization, the resulting vector of the calculated cross-correlation is limited to $\{c \in \mathbb{R} \mid-1 \leq c \leq 1\}$. De-

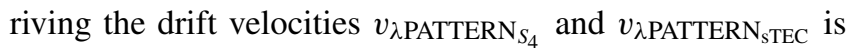
straightforward when the distance of both measurement locations, $s_{\mathrm{IPP}}$, is known:

$v_{\lambda_{\text {PATTERN }_{S_{4}}}}=\frac{s_{\text {IPP }}}{\tau_{S_{4}}} \quad v_{\lambda \text { PATTERN }_{\text {STEC }}}=\frac{s_{\text {IPP }}}{\tau_{\text {STEC }}}$.

To reconstruct the link geometry, the satellite coordinates are calculated according to IS-GPS200H (2013) by using the received broadcast ephemeris data and the antenna positions of both stations as given in Table 3. In this analysis the observation location is defined to be the location of the IPP by using a single-shell approximation for the ionosphere at the assumed plasma irregularity height, $h_{\mathrm{I}}$. The latitude, $\varphi_{\mathrm{IPP}_{i}}$, and longitude, $\lambda_{\mathrm{IPP}_{i}}$, of the IPP from both receivers to the same GNSS satellite link can be derived for every time step from the GNSS link geometry by applying the following equations according to Klobuchar (1987): 


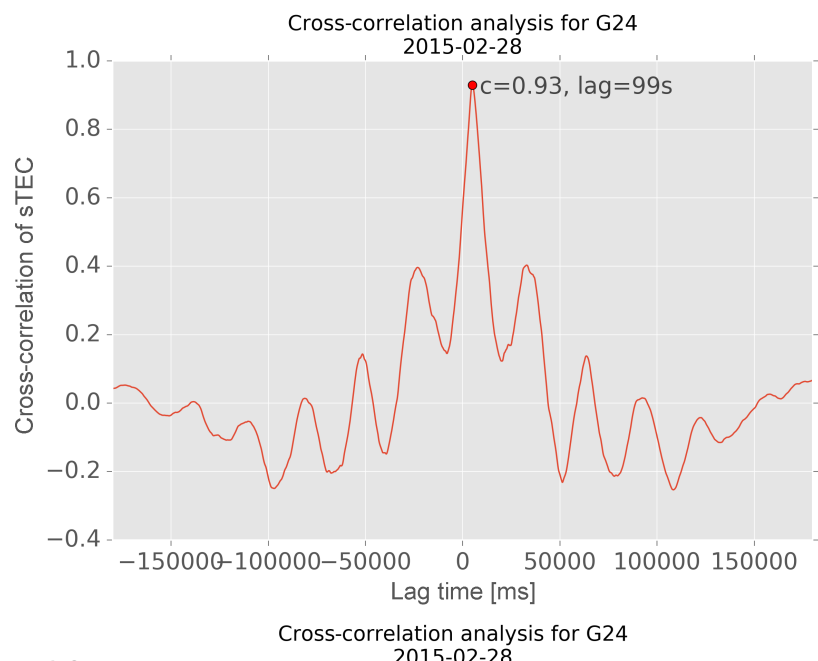

Table 3. Locations of DLR's high-rate GNSS stations in Bahir Dar, Ethiopia.

\begin{tabular}{lrr}
\hline & msbd01 & msbd02 \\
\hline$X(\mathrm{~m})$ & 4966228 & 4970637 \\
$Y(\mathrm{~m})$ & 3796382 & 3790751 \\
$Z(\mathrm{~m})$ & 1271522 & 1271341 \\
\hline Long $\left(^{\circ} \mathrm{E}\right)$ & $37^{\circ} 23^{\prime} 45^{\prime \prime}$ & $37^{\circ} 19^{\prime} 49^{\prime \prime}$ \\
Lat $\left({ }^{\circ} \mathrm{N}\right)$ & $11^{\circ} 34^{\prime} 23^{\prime \prime}$ & $11^{\circ} 34^{\prime} 16^{\prime \prime}$ \\
Alt $(\mathrm{m})$ & 1800 & 1850 \\
\hline
\end{tabular}

Reference system is WGS84.

The mean zonal speed of both IPPs can be calculated using the zonal IPP velocity of both stations:

$$
\begin{aligned}
v_{\lambda \mathrm{IPP}} & =\left(R_{\mathrm{E}}+h_{\mathrm{I}}\right) \cdot \frac{1}{2}\left(\frac{\Delta \lambda_{\mathrm{IPP}_{1}}}{\Delta t} \cos \varphi_{\mathrm{IPP}_{1}}\right. \\
& \left.+\frac{\Delta \lambda_{\mathrm{IPP}_{2}}}{\Delta t} \cos \varphi_{\mathrm{IPP}_{2}}\right) .
\end{aligned}
$$

For the observed GPS satellite G24 the mean distance $s_{\text {IPP }}$ according to Eq. (12) is about $7 \mathrm{~km}$. For a lag time of approximately $99 \mathrm{~s}$, the estimated approximative pattern speed is $v_{\lambda \text { PATTERN }}=71 \mathrm{~m} \mathrm{~s}^{-1}$. Since G24 was mostly moving from north to south, a very low mean zonal velocity of both IPPs was observed; $v_{\lambda I P P}=10 \mathrm{~m} \mathrm{~s}^{-1}$. Considering the IPP movement according to Eq. (4) and neglecting effects of upward drifts, we have computed a plasma irregularity velocity of $v_{\lambda}=81 \mathrm{~m} \mathrm{~s}^{-1}$. These drift velocities agree quite well with those derived by Wiens et al. (2006). The zonal dimension of the irregularity region is assumed to be $292 \mathrm{~km}$.

As shown in Fig. 10, we observed an interesting behaviour for GPS link G29. Beginning at 23:10 LT (20:10 UTC), we observed a depletion signature moving eastward with a velocity of approximately $80 \mathrm{~m} \mathrm{~s}^{-1}$. After midnight the eastward propagation decreased and finally rapidly changed to a depletion signature moving westward with a velocity of approximately $102 \mathrm{~m} \mathrm{~s}^{-1}$. Kil et al. (2000), Bhattacharyya et al. (2002) and Abdu et al. (2003) observed similar characteristics while analysing GPS observations, geostationary satellite observations at Ancón, Peru, and optical measurements $(630 \mathrm{~nm})$ at Cachoeira Paulista, Brazil. The authors concluded that these results point to the dominant role of a disturbance-dynamo-associated westward thermospheric wind. This explanation might be applicable to our observations when looking at the enhanced $\mathrm{Kp}$ index plotted in Fig. 11. Under geomagnetic quiet conditions a reversal of the drift was found to be much smoother with a reversal time during early morning around 04:00 to 05:00 LT (e.g. Fejer et al., 1985, 1991).

The presented results of plasma irregularity characteristics at low latitudes (Table 4) underline previous studies of Kil et al. (2000), Ledvina et al. (2004), Wiens et al. (2006), Yao 

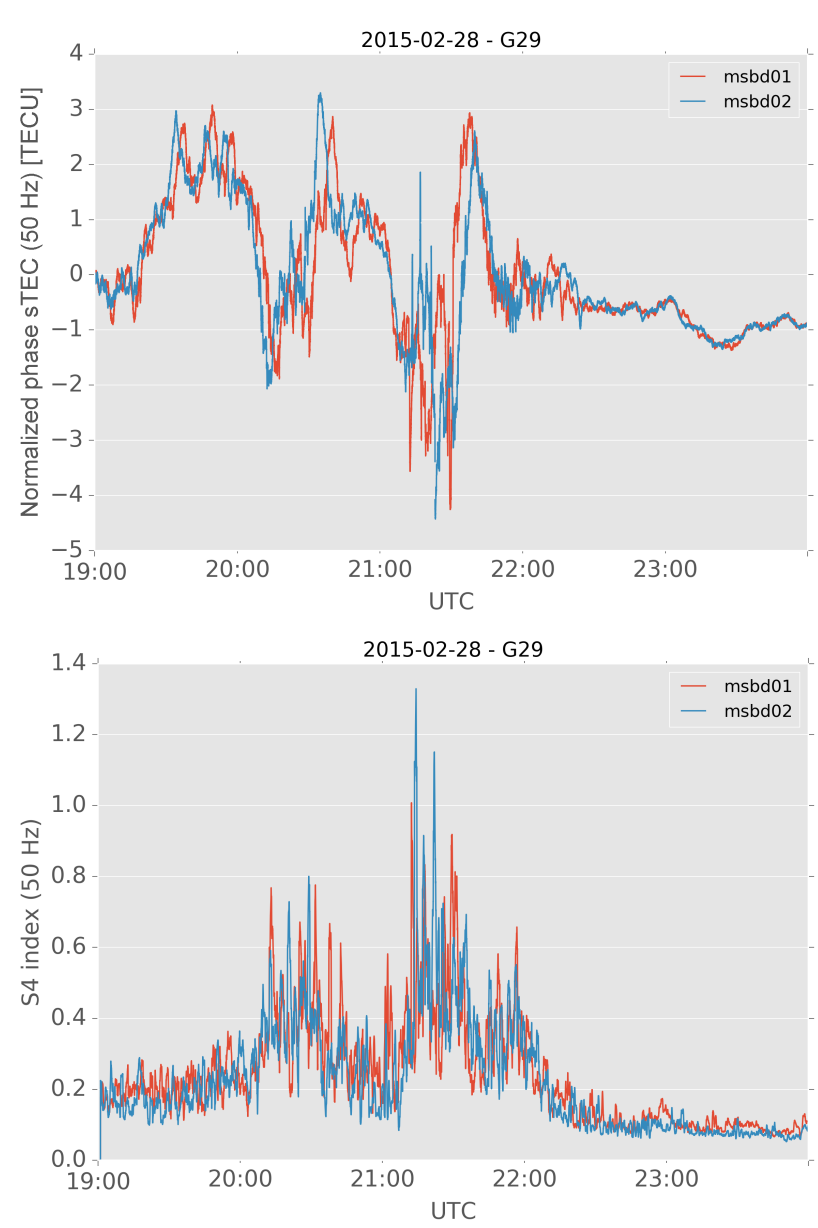

Figure 10. Normalized sTEC and $S_{4}$ measurements for G29 used as input for the cross-correlation analysis showing a reversal of the drift direction after midnight local time (21:00 UTC).

and Makela (2007) and Ji et al. (2011) that reported plasma drift velocities around midnight between 50 and $150 \mathrm{~m} \mathrm{~s}^{-1}$ and a zonal extension up to approximately $500 \mathrm{~km}$.

\section{Conclusions}

This paper presents a preliminary investigation of scintillation measurements during the high solar activity phase of solar cycle 24 in the low-latitude region at Bahir Dar, Ethiopia. To capture the small-scale irregularities over Bahir Dar's ionosphere, we have implemented the spaced-receiver method by employing two high-rate GNSS receiver stations (msbd01 and msbd01). Small-scale ionospheric irregularities are common phenomena in the low-latitude ionosphere after sunset and could be properly recorded by high-rate GPS measurements. We observed eastward drifting plasma irregularities after sunset by monitoring sTEC depletions and enhancements of radio scintillation activity. By applying the cross-correlation analysis method we could derive zonal ir-

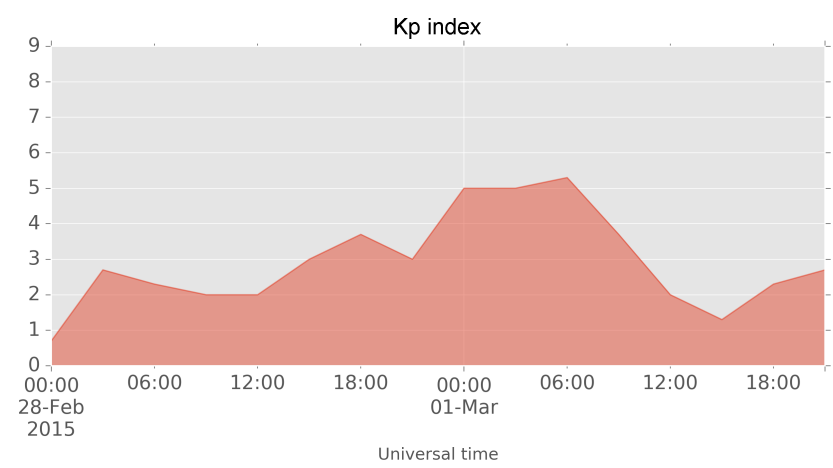

Figure 11. The night on 1 March belongs to the five most disturbed days in March 2015 with a Kp Index > 5 (GFZ, 2010).

Table 4. Plasma irregularity characterization over Bahir Dar, Ethiopia.

\begin{tabular}{lrllrr}
\hline Date & LT & PRN & Direction & Velocity & Size \\
\hline 28 Feb 2015 & $23: 30$ & G24 & eastward & $81 \mathrm{~m} \mathrm{~s}^{-1}$ & $292 \mathrm{~km}$ \\
28 Feb 2015 & $23: 10$ & G29 & eastward & $80 \mathrm{~m} \mathrm{~s}^{-1}$ & $144 \mathrm{~km}$ \\
28 Feb 2015 & $24: 10$ & G29 & westward & $102 \mathrm{~m} \mathrm{~s}^{-1}$ & $312 \mathrm{~km}$ \\
\hline 8 Apr 2015 & $23: 00$ & G21 & eastward & $80 \mathrm{~m} \mathrm{~s}^{-1}$ & $58 \mathrm{~km}$ \\
8 Apr 2015 & $23: 00$ & G26 & eastward & $84 \mathrm{~m} \mathrm{~s}^{-1}$ & $151 \mathrm{~km}$ \\
8 Apr 2015 & $23: 30$ & G26 & eastward & $78 \mathrm{~m} \mathrm{~s}^{-1}$ & $187 \mathrm{~km}$ \\
\hline
\end{tabular}

regularity drift velocities of about $80 \mathrm{~m} \mathrm{~s}^{-1}$ eastward and under enhanced geomagnetic disturbances even a westward reversal. This supports results of prior investigations when analysing data from geostationary satellites and optical instruments. Several research institutions in this research field such as Boston College, DLR, IEEA, Kyoto University, TUB and the United States Air Force (USAF), have been deploying ground receivers/sensors in Bahir Dar, Ethiopia. Thus, coordinated measurements of these different receivers will enable studying spatial characteristics and dynamics of the irregularity pattern in more detail. Future studies will utilize an extended network of high-rate GNSS stations with its multi-constellation links. Combining the ground-based GNSS measurements with spaceborne observations such as ESA's Swarm constellation mission or regional beacon measurements, the increasing scientific opportunities in Africa can significantly improve our understanding of the spatial and temporal characteristics of plasma irregularities at low latitudes.

\section{Data availability}

The high-rate GNSS raw data used in this study cover the Ethiopian region around Bahir Dar and are stored in an internal DLR archive. Direct open access to this archive is not possible, since data stored there are used for other research activities too. However, access to the DLR GNSS raw data 
used within this study can be requested by contacting the first author of this paper.

Acknowledgements. The authors appreciate the support from Bahir Dar University for hosting and maintaining both GNSS stations within the framework agreement between DLR and BDU. The authors also thank Roman Galas and Maria Cokrlic from the Technical University Berlin for providing $S_{4}$ data used within this study.

The article processing charges for this open-access publication were covered by a Research

Centre of the Helmholtz Association.

The topical editor, C. Stolle, thanks Y. Beniguel and two anonymous referees for their help in evaluating this paper.

\section{References}

Aarons, J.: Global morphology of ionospheric scintillations, IEEE Proc., 70, 360-378, doi:10.1109/PROC.1982.12314, 1982.

Abdu, M. A., Batista, I. S., Takahashi, H., MacDougall, J., Sobral, J. H., Medeiros, A. F., and Trivedi, N. B.: Magnetospheric disturbance induced equatorial plasma bubble development and dynamics: A case study in Brazilian sector, J. Geophys. Res.-Space, 108, 1449, doi:10.1029/2002JA009721, 2003.

Alfonsi, L., Spogli, L., Pezzopane, M., Romano, V., Zuccheretti, E., De Franceschi, G., Cabrera, M. A., and Ezquer, R. G.: Comparative analysis of spread-F signature and GPS scintillation occurrences at Tucumán, Argentina, J. Geophys. Res.-Space, 118, 4483-4502, doi:10.1002/jgra.50378, 2013.

Basu, S. and Basu, S.: Equatorial scintillations - a review, J. Atmos. Terr. Phys., 43, 473-489, doi:10.1016/0021-9169(81)90110-0, 1981.

Bhattacharyya, A., Basu, S., Groves, K. M., Valladares, C. E., and Sheehan, R.: Effect of magnetic activity on the dynamics of equatorial F region irregularities, J. Geophys. Res.-Space, 107, 1489, doi:10.1029/2002JA009644, 2002.

Briggs, B.: On the analysis of moving patterns in geophysics I. Correlation analysis, J. Atmos. Terr. Phys., 30, 1777-1788, doi:10.1016/0021-9169(68)90097-4, 1968.

Briggs, B. H., Phillips, G. J., and Shinn, D. H.: The Analysis of Observations on Spaced Receivers of the Fading of Radio Signals, P. Phys. Soc. B, 63, 106, doi:10.1088/0370-1301/63/2/305, 1950.

Čokrlić, M. and Galas, R.: TUB software tools for monitoring ionospheric irregularities in a single station mode and first results, in: Antennas and Propagation (EuCAP), 2013 7th European Conference, 3673-3676, 2013.

Fejer, B. G., Kudeki, E., and Farley, D. T.: Equatorial F region zonal plasma drifts, J. Geophys.Res.-Space, 90, 12249-12255, doi:10.1029/JA090iA12p12249, 1985.

Fejer, B. G., de Paula, E. R., González, S. A., and Woodman, R. F.: Average vertical and zonal F region plasma drifts over Jicamarca, J. Geophys. Res.-Space, 96, 13901-13906, doi:10.1029/91JA01171, 1991.

GFZ: International Q-Days and D-Days since 2010, available at: http://www.gfz-potsdam.de/en/section/earths-magnetic-field/ data-products-services/kp-index/qd-days/qd-days-since-2010/ (last access: 16 December 2016), 2010.

Hargreaves, J. K.: The Solar-Terrestrial Environment, Cambridge University Press, doi:10.1017/CBO9780511628924, Cambridge Books Online, 1992.

Hegarty, C., El-Arini, M. B., Kim, T., and Ericson, S.: Scintillation modeling for GPS-Wide Area Augmentation System receivers, Radio Sci., 36, 1221-1231, doi:10.1029/1999RS002425, 2001.

Hlubek, N., Berdermann, J., Wilken, V., Gewies, S., Jakowski, N., Wassaie, M., and Damtie, B.: Scintillations of the GPS, GLONASS, and Galileo signals at equatorial latitude, J. Space Weather Space Clim., 4, A22, doi:10.1051/swsc/2014020, 2014.

Hofmann-Wellenhof, B., Lichtenegger, H., and Collins, J.: Global Positioning System - Theory and Practice, Springer-Verlag Wien, doi:10.1007/978-3-7091-6199-9, 2001.

IS-GPS200H: GLOBAL POSITIONING SYSTEMS DIRECTORATE ENGINEERING \& INTEGRATION INTERFACE SPECIFICATION IS-GPS200H, available at: http://www.gps. gov/technical/icwg/IS-GPS-200H.pdf (last access: 16 December 2016), 2013.

Jakowski, N., Mayer, C., Hoque, M. M., and Wilken, V.: Total electron content models and their use in ionosphere monitoring, Radio Sci., 46, rS0D18, doi:10.1029/2010RS004620, 2011.

JAVAD: GREIS - GNSS Receiver External Interface Specification, available at: http://www.javad.com/downloads/javadgnss/ manuals/GREIS/GREIS_Reference_Guide.pdf (last access: 16 December 2016), 2015.

Ji, S., Chen, W., Ding, X., and Zhao, C.: Equatorial ionospheric zonal drift by monitoring local GPS reference networks, J. Geophys. Res.-Space, 116, a08310, doi:10.1029/2010JA015993, 2011.

Kelley, M.: The Earth's Ionosphere: Plasma Physics and Electrodynamics, International Geophysics, Elsevier Science, USA and UK, 2009.

Kil, H., Kintner, P. M., de Paula, E. R., and Kantor, I. J.: Global Positioning System measurements of the ionospheric zonal apparent velocity at Cachoeira Paulista in Brazil, J. Geophys. Res.Space, 105, 5317-5327, doi:10.1029/1999JA000244, 2000.

Klobuchar, J. A.: Ionospheric Time-Delay Algorithm for SingleFrequency GPS Users, IEEE T. Aero. Elec. Sys., AES-23, 325331, doi:10.1109/TAES.1987.310829, 1987.

Ledvina, B. M., Kintner, P. M., and Makela, J. J.: Temporal properties of intense GPS L1 amplitude scintillations at midlatitudes, Radio Sci., 39, rS1S18, doi:10.1029/2002RS002832, 2004.

Muella, M. T. A. H., Kherani, E. A., de Paula, E. R., Cerruti, A. P., Kintner, P. M., Kantor, I. J., Mitchell, C. N., Batista, I. S., and Abdu, M. A.: Scintillation-producing Fresnel-scale irregularities associated with the regions of steepest TEC gradients adjacent to the equatorial ionization anomaly, J. Geophys. Res.-Space, 115, A03301, doi:10.1029/2009JA014788, 2010.

Noack, T., Engler, E., and Klähn, D.: High Rate Performance, Assessment of GNSS Raw Data Based on the DLR Experimentation and Verification Network, in: ION GNSS 2005, September, Long Beach, USA, 2005.

Subirana, J. S., Zornoza, J. J., and Hernández-Pajares, M.: GNSS DATA PROCESSING: Volume I: Fundamentals and Algorithms (ESA TM-23/1, May 2013), ESA Communications ESTEC, the Netherlands, 2013. 
Valladares, C. E., Villalobos, J., Sheehan, R., and Hagan, M. P.: Latitudinal extension of low-latitude scintillations measured with a network of GPS receivers, Ann. Geophys., 22, 3155-3175, doi:10.5194/angeo-22-3155-2004, 2004.

Wang, J. and Morton, Y. T.: High-Latitude Ionospheric Irregularity Drift Velocity Estimation Using Spaced GPS Receiver Carrier Phase Time-Frequency Analysis, IEEE T. Geosci. Remote Sens., 53, 6099-6113, doi:10.1109/TGRS.2015.2432014, 2015.
Wiens, R. H., Ledvina, B. M., Kintner, P. M., Afewerki, M., and Mulugheta, Z.: Equatorial plasma bubbles in the ionosphere over Eritrea: occurrence and drift speed, Ann. Geophys., 24, 14431453, doi:10.5194/angeo-24-1443-2006, 2006.

Yao, D. and Makela, J. J.: Analysis of equatorial plasma bubble zonal drift velocities in the Pacific sector by imaging techniques, Ann. Geophys., 25, 701-709, doi:10.5194/angeo-25-701-2007, 2007. 